\title{
Emprego da Ultra-Sonografia Cervical no Seguimento de Pacientes Com Carcinoma Diferenciado de Tireóide
}

\begin{abstract}
RESUMO
A ultra-sonografia (US) cervical é recomendada na avaliação de todos pacientes com carcinoma de tireóide após a terapia inicial, pois mesmo indivíduos de baixo risco com tireoglobulina $(\mathrm{Tg})$ estimulada indetectável podem apresentar metástases cervicais. Para estas metástases, a US é o método mais sensível, superior à pesquisa de corpo inteiro (PCl) com ${ }^{131}$ I. Linfonodos cervicais com diâmetro $\geq 5 \mathrm{~mm}$ com calcificações finas e/ou degeneração cística quase sempre são de etiologia maligna. $\mathrm{Na}$ ausência destas características, o formato arredondado e a ausência do hilo ecogênico são achados "suspeitos", enquanto linfonodos alongados e com hilo ecogênico visível são considerados benignos. A avaliação do fluxo, através do doppler, auxilia no diagnóstico diferencial, usualmente revelando hipervascularização periférica ou mista nos casos malignos. Na presença de linfonodos "suspeitos" na US, a avaliação citológica do material obtido através da punção aspirativa por agulha fina (PAAF) e a dosagem da Tg, obtida do lavado da agulha, são testes úteis e complementares para definir a etiologia, com elevada sensibilidade quando combinados e especificidade de $100 \%$. A US também é útil antes da tiroidectomia, auxiliando e até, em alguns casos, modificando o planejamento cirúrgico; e antes da ablação, para mensuração dos remanescentes tireoidianos e pesquisa de metástases linfonodais persistentes. Outra aplicação desse método de imagem é guiar a injeção de etanol (escleroterapia) ou a introdução de eletrodos para ablação com radiofreqüência em casos selecionados de metástases linfonodais isoladas, como alternativa às terapias convencionais. (Arq Bras Endocrinol Metab 2007;51/4:593-600)
\end{abstract}

Descritores: Linfonodos; Ultra-sonografia; Carcinoma de tireóide; Citologia aspirativa; Tireoglobulina

\section{ABSTRACT}

\begin{abstract}
Usefulness of Neck Ultrasonography in the Follow-Up of Patients With Differentiated Thyroid Cancer.

Neck ultrasonography (US) is recommended for the assessment of all patients with thyroid carcinoma after initial therapy, since even low-risk patients with undetectable stimulated thyroglobulin ( $\mathrm{Tg}$ ) may present cervical metastases. In the case of these metastases, US is the most sensitive method and is superior to whole-body ${ }^{131}$ I scanning. Cervical lymph nodes with a diameter $\geq 5 \mathrm{~mm}$ presenting thin calcifications and/or cystic degeneration have almost always a malignant etiology. In the absence of these characteristics, a round shape and the absence of an echogenic hilum are "suspicious" findings, whereas elongated lymph nodes with a visible echogenic hilum are considered benign. Doppler flow analysis helps with the differential diagnosis, usually revealing peripheral or mixed hypervascularization in malignant cases. In the presence of "suspicious" lymph nodes upon US, fine-needle aspiration cytology and measurement of $\mathrm{Tg}$ in the needle lavage fluid are useful and complementary exams for the definition of the etiology, with the combination of the two methods showing elevated sensitivity and $100 \%$ specificity. US is also useful before thyroidectomy, even contributing in some cases to modify the surgical planning, and before ablation for the measurement of thyroid remnants and detection of persistent lymph node metastases. Another application of this imaging method is to guide the injection of ethanol (sclerotherapy) or the introduction of electrodes for radiofrequency ablation in selected cases of isolated lymph node metastases as an alternative to traditional therapies. (Arq Bras Endocrinol Metab 2007;51/4:593-600)
\end{abstract}

Keywords: Lymph nodes; Ultrasonography; Thyroid carcinoma; Aspiration cytology; Thyroglobulin

\section{artigo original}

\author{
PEDRo W. Rosário \\ WILSON C. TAVARES JÚNIOR \\ Rosa paula M. Biscolla \\ SAULO PuRISCH
}

RUI M.B. MACIEL

Serviço de Endocrinologia da Santa Casa de Belo Horizonte (PWR \& SP) e Instituto Alpha de Gastroenterologia (WCTJ), Belo Horizonte, MG; e Disciplina de Endocrinologia (RPMB \& RMBM), Departamento de Medicina, Escola Paulista de Medicina/ Universidade Federal de São Paulo (EPM/UNIFESP), São Paulo, SP.

Recebido em 22/09/06

Revisado em 05/10/06

Aceito em 15/12/06 
$\mathrm{N}$ O SEGUIMENTO DE PACIENTES com câncer diferenciado de tireóide, destacam-se alguns avanços, como a melhora da sensibilidade dos ensaios da tireoglobulina sérica (sTg), a elaboração de protocolos específicos para pacientes de baixo risco e a disponibilidade do TSH recombinante (rhTSH) (1-3).

Recentemente, o uso da ultra-sonografia (US) cervical tem assumido papel importante no seguimento dos pacientes com carcinoma de tireóide, permitindo a detecção precoce de metástases em linfonodos, muitas vezes não palpáveis. A importância da US se baseia na observação de que em pacientes com metástases cervicais, 20-40\% destas não captam ${ }^{131} \mathrm{I}$, mesmo após a administração de altas doses desse rádio-fármaco (4); além disso, percentual semelhante de pacientes cursam com dosagens indetectáveis de $\mathrm{sTg}$, mesmo em vigência de níveis séricos elevados de TSH (5-8).

Atualmente, a US cervical é recomendada de rotina no seguimento de pacientes com câncer de tireóide $(1,2,9)$, sendo importante o conhecimento de sua contribuição, do significado dos achados ultrasonográficos, suas limitações e das perspectivas de aplicações deste método de imagem. Neste artigo, esses aspectos foram revisados.

\section{NECESSIDADE DA US NO SEGUIMENTO DE PACIENTES COM CÂNCER DE TIREÓIDE}

Baseado nos dados de cinco séries recentes $(5,6,10-12)$ que avaliaram pacientes, na maioria de baixo risco tratados com tireoidectomia total e ${ }^{131} \mathrm{I}$, verificamos que 8.431 pacientes $(1,8 \%)$, apesar da sTg indetectável após suspensão de L-T4, exibiam linfonodos suspeitos na US cervical, posteriormente confirmados como metástases $(5,10)$, assim como 7.398 pacientes $(1,7 \%)$ com sTg indetectável após estímulo com rhTSH $(6,11,12)$ (tabela 1). Portanto, mesmo em pacientes de baixo risco com sTg estimulada indetectável após a terapia com ${ }^{131}$ I é possível encontrar metástases linfonodais.

Dados de 4 estudos (5-8) nos quais a US foi realizada de rotina no seguimento após tireoidectomia total e ablação com ${ }^{131} \mathrm{I}$, mostram que a sensibilidade desse método para metástases cervicais variou de 70 a $100 \%$, enquanto que a da sTg estimulada variou de 57 a $82 \%$ e da PCI, de 20 a 58\%. Em 169 pacientes com doença cervical, 90\% apresentaram US positiva, enquanto que a PCI detectou apenas $42 \%$ dos casos (5-8) (tabela 2). Portanto, podemos concluir que a US é o método mais sensível para detecção de metástases cervicais, identificando lesões geralmente não visualizadas pela PCI e sendo recomendada de rotina no nosso meio, independentemente do risco ou nível de sTg $(9,13)$.

Apesar de a maioria dos estudos relatar a utilidade da US no seguimento dos pacientes após tireoidectomia total e terapia com ${ }^{131} \mathrm{I}$, a realização deste exame previamente à cirurgia tem se mostrado muito útil, podendo revelar metástases não palpáveis, auxiliar na definição e até, em alguns casos, modificar o planejamento cirúrgico (14). Essa conduta determina uma maior chance de ressecção completa de linfonodos metastáticos que poderiam não ser detectados sem a realização prévia da US. Além disso, metástases cervicais não ressecadas durante a cirurgia podem não ser visualizadas na PCI pós-dose e cursarem com Tg pós-operatória baixa, sendo detectados apenas pela US (15). Estudo recente mostrou que a sensibilidade da US para metástases cervicais persistentes após a cirurgia inicial e não palpáveis foi de $94 \%$, sendo superior à PCI pós-dose (sensibilidade 71\%) (15).

O emprego da US cervical previamente à dose ablativa de ${ }^{131} \mathrm{I}$ apresenta papel importante nos paci-

Tabela 1. Freqüência de metástases cervicais em pacientes de baixo risco com Tg sérica estimulada indetectável após a terapia inicial.

\begin{tabular}{lccc}
\hline Estudo (referência) & Pacientes & $\begin{array}{c}\text { Valor de corte da } \\
\text { Tg sérica }\end{array}$ & $\begin{array}{c}\text { Frequiência de metástases } \\
\text { cervicais }\end{array}$ \\
Torlontano e cols. 2004 (5) & 335 & $1 \mathrm{ngmL}(\mathrm{sem} T 4)$ & $2 \%$ \\
Rosario e cols. 2005 (10) & 96 & $1 \mathrm{ngmL}(\mathrm{sem} \mathrm{T4)}$ & $1 \%$ \\
David e cols. 2005 (11) & 70 & $1 \mathrm{ngmL}(\mathrm{rhTSH})$ & $1,4 \%$ \\
Torlontano e cols. 2003 (12) & 78 & $1 \mathrm{ngmL}(\mathrm{rhTSH})$ & $2,5 \%$ \\
Pacini e cols. 2003 (6) & 250 & $1 \mathrm{ngmL}(\mathrm{rhTSH})$ & $1,6 \%$ \\
\hline
\end{tabular}

Tabela 2. Sensibilidade dos métodos diagnósticos tradicionais para metástases cervicais.

\begin{tabular}{lcccc}
\hline Estudo (referência) & Pacientes & $\begin{array}{c}\text { Tg sérica } \\
\text { estimulada }\end{array}$ & PCI & US \\
Torlontano e cols. 2004 (5) & 38 & $81,6 \%$ & $34,2 \%$ & $100 \%$ \\
Pacini e cols. 2003 (6) & 30 & $78 \%$ & $20 \%$ & $70 \%$ \\
Rosario e cols. 2004 (7) & 50 & $66 \%$ & $58 \%$ & $96 \%$ \\
Frasoldati e cols. 2003 (8) & 51 & $56,8 \%$ & $45 \%$ & $94 \%$ \\
\hline
\end{tabular}


entes cuja cirurgia foi realizada em outro serviço ou onde não seja possível determinar a extensão da tireoidectomia ou se houve pesquisa de metástases linfonodais per-operatória. Nestes pacientes, a US pode detectar grandes remanescentes tireoidianos ou linfonodos metastáticos, apontando para a necessidade de nova intervenção cirúrgica antes da administração do 131I (16).

As indicações da US em pacientes com carcinoma diferenciado de tireóide encontram-se na tabela 3 .

\section{DIFERENCIAÇÃO ENTRE LINFONODOS CERVICAIS MALIGNOS E BENIGNOS}

Na região cervical, comumente encontram-se linfonodomegalias reacionais a processos inflamatórios inespecíficos e, mais raramente, secundários a doenças granulomatosas como tuberculose ou linfonodos acometidos por neoplasias linfoproliferativas ou por metástases de outros tumores. É importante que, no paciente com carcinoma de tireóide (principalmente carcinoma papilífero), uma avaliação criteriosa seja feita na presença de linfonodos na US. Discutiremos, a seguir, o diagnóstico diferencial da linfadenopatia cervical nesses pacientes.

\section{Diferenciação ultra-sonográfica}

Baseado na experiência da Santa Casa de Belo Horizonte em 112 pacientes com câncer papilífero, que tiveram as características ultra-sonográficas de seus linfonodos correlacionadas com a histologia (17), propomos a seguinte classificação para os linfonodos cervicais com diâmetro $\geq 5 \mathrm{~mm}$ :

Grau I (figura 1): sem calcificações ou degeneração cística, com hilo ecogênico visível e forma alongada (maior menor diâmetro $>2$ ). Nenhum linfonodo maligno e $65,4 \%$ dos benignos exibiram estes achados.
Grau II (figura 2): sem calcificações ou degeneração cística, sem hilo ecogênico aparente ou com forma arredondada (presença de apenas uma das últimas características). Oito por cento dos linfonodos malignos e $30,7 \%$ dos benignos estavam neste grupo.

Grau III (figura 3): sem calcificações ou degeneração cística, forma arredondada e hilo ecogênico ausente (presença das duas últimas características). Estes achados foram vistos em $24 \%$ dos linfonodos malignos e em 3,8\% dos benignos.

Grau IV (figura 4): com calcificações finas e/ou degeneração cística. $68 \%$ dos linfonodos malignos exibiram estas características, nenhum linfonodo reacional.

Considerando linfonodos grau I e II como benignos, e III e IV como malignos, encontra-se uma sensibilidade de $92 \%$ e especificidade de 96\% (17) (tabela 4). Essa sensibilidade é maior quando se investiga alguns linfonodos grau II.

Calcificações finas são comuns em metástases de carcinoma papilífero, variando de 50 a 69\% (17-19) e raras em outras neoplasias. Essa característica não é vista em linfonodos reacionais a processos inespecíficos, mas pode estar presente em linfonodos após radioterapia ou tuberculose (calcificações grosseiras). A presença de áreas de degeneração cística (18-20) constitui achado com alta especificidade para malignidade ou tuberculose. A ausência de hilo ecogênico combinado à forma arredondada identifica a maioria dos linfonodos metastáticos sem calcificações ou áreas císticas $(17,21)$.

A dopplerfluxometria de linfonodos malignos geralmente revela hipervascularização (periférica) ou mista (periférica e central) $(5,8,12)$, enquanto que o índice de resistência ou resistividade, usualmente elevado em outras metástases, pode ser baixo nas de carcinoma papilífero $(22)$.

Tabela 3. Indicações da US em pacientes com câncer diferenciado de tireóide.

\begin{tabular}{|c|c|c|}
\hline Momento da avaliação & Casos indicados & Objetivo \\
\hline Antes da cirurgia inicial & $\begin{array}{l}\text { Diagnóstico pré-operatório de } \\
\text { câncer ou altamente suspeito }\end{array}$ & $\begin{array}{l}\text { Pesquisa de multicentricidade, metástases } \\
\text { cervicais e invasão tumoral extensa }\end{array}$ \\
\hline $\begin{array}{l}\text { Após uma lobectomia } \\
\text { (microcarcinoma único) }\end{array}$ & Todos, anualmente & $\begin{array}{l}\text { Pesquisa de metástases cervicais e/ou } \\
\text { recorrência em lobo remanescente }\end{array}$ \\
\hline $\begin{array}{l}\text { Após a tireoidectomia total } \\
\text { (antes da ablação com }{ }^{131} \text { I) }\end{array}$ & $\begin{array}{l}\text { Todos, especialmente com } \\
\text { metástases linfonodais na cirurgia }\end{array}$ & $\begin{array}{l}\text { Mensuração de remanescentes tireoidianos } \\
\text { e pesquisa de metástases cervicais persistentes }\end{array}$ \\
\hline $\begin{array}{l}6-12 \text { meses após a } \\
\text { tireoidectomia e } 131 \mid\end{array}$ & $\begin{array}{l}\text { Todos, independentemente do } \\
\text { risco e nível da sTg }\end{array}$ & \\
\hline Seguimento tardio & $\begin{array}{l}\text { Alto risco e/ou com sTg } \\
\text { detectável }\end{array}$ & \\
\hline Recidiva cervical & $\begin{array}{l}\text { Pacientes de baixo risco, com } \\
\text { metástases linfonodais isoladas }\end{array}$ & $\begin{array}{l}\text { Orientar a escleroterapia com etanol, como } \\
\text { alternativa ao radioiodo e à cirurgia }\end{array}$ \\
\hline
\end{tabular}




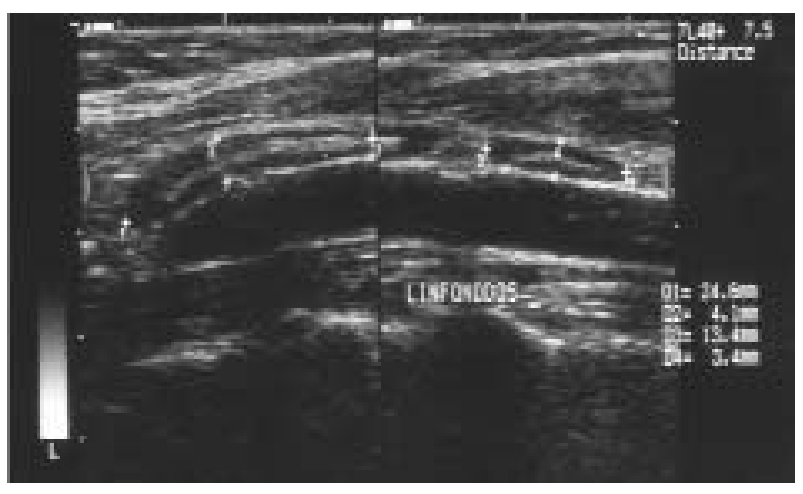

Figura 1. Linfonodo grau I.

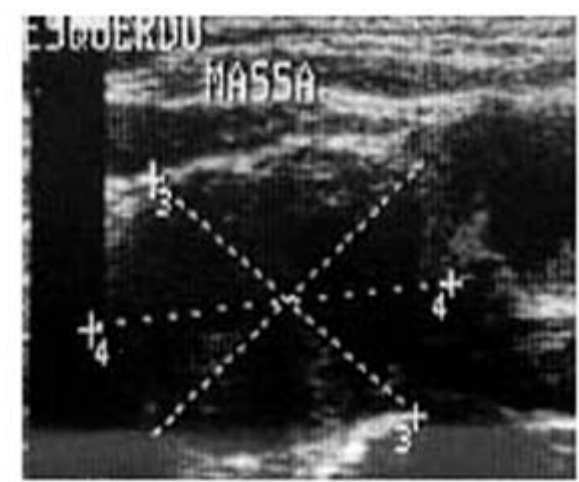

Figura 3. Linfonodo grau III.

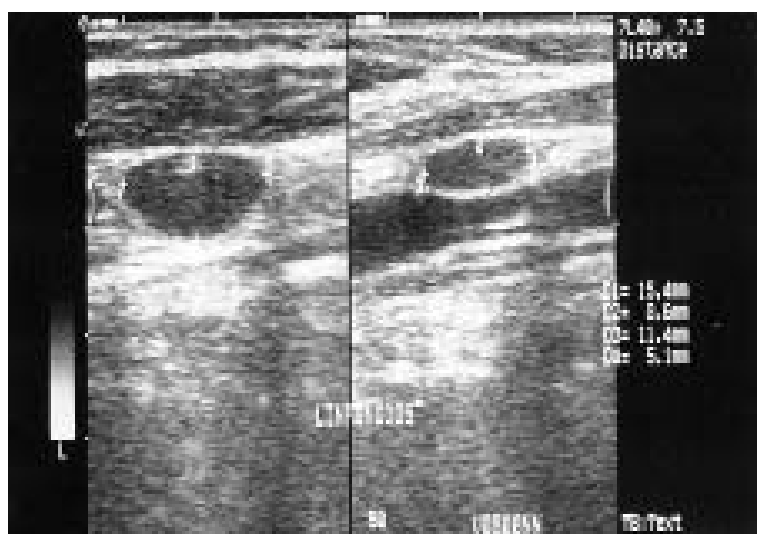

Figura 2. Linfonodo grau II.

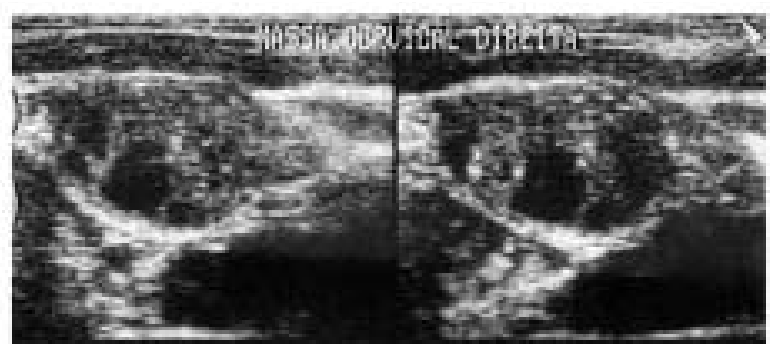

Figura 4. Linfonodo grau IV.

Tabela 4. Sensibilidade e especificidade da combinação de características ultra-sonográficas de linfonodos cervicais $\geq 5 \mathrm{~mm}$ em pacientes com carcinoma papilífero (17).

\begin{tabular}{lcc}
\hline Benigno / Maligno & Sensibilidade & Especificidade \\
Grau I / Grau II, III, IV & $100 \%$ & $65,4 \%$ \\
Grau I, II / Grau III, IV & $92 \%$ & $96 \%$ \\
Grau I, II, III / Grau IV & $68 \%$ & $100 \%$ \\
\hline
\end{tabular}

\section{Conduta nos casos com linfonodos suspeitos de malignidade na US}

Após a detecção de linfonodos "suspeitos" à US, a avaliação citológica do material obtido através da punção aspirativa por agulha fina (PAAF) é necessária e de fundamental importância para definição etiológica da linfonodomegalia (11,23-27), principalmente em pacientes com sTg indetectável e/ou metástases não iodo-captantes. Nos casos em que a PCI também foi realizada, a presença de captação na topografia correspondente tem elevada especificidade, sendo suficiente para confirmar a natureza metastática do linfonodo, mas os pacientes podem apresentar mais de um linfonodo acometido na região cervical e nem todos serem iodocaptantes.
Os resultados mais freqüentemente encontrados com a realização da PAAF são: "linfonodo reacional", quando a citologia mostra presença de linfócitos em diferentes estágios de maturação, "linfonodo metastático", quando à citologia encontram-se células foliculares em substituição à população linfocitária, ou "não diagnóstica", quando o estudo citológico é prejudicado pela insuficiência de material obtido. Menos freqüentemente, podemos encontrar achados compatíveis com doenças granulomatosas (tuberculose, sarcoidose), com neoplasias como linfomas ou metástases de outros carcinomas (laringe, pulmão ou mama) $(23,26,28)$. Em 32 pacientes com carcinoma papilífero tratados com tireoidectomia total e ${ }^{131}$ I e em segui- 
mento no ambulatório de tireóide da EPM/UNIFE$\mathrm{SP}$, os resultados da PAAF, realizada em 44 linfonodos visualizados à US cervical, foram "linfonodos reacionais" em 65\%, "linfonodos metastáticos" em 29\% e material "não diagnóstico" em 6\% (27). Este último dado está de acordo com a literatura, que refere resultado não diagnóstico em $8 \%$ das citologias $(20,29,30)$.

Nos últimos anos, a dosagem da $\mathrm{Tg}$, obtida do lavado da agulha utilizada na realização da PAAF (TgPAAF), tem sido proposta para diferenciar linfonodos "reacionais" de "metastáticos". Pacini e cols. foram os primeiros a demonstrar, em 1992, níveis elevados de Tg-PAAF em linfonodos com metástases de carcinoma de tireóide, em oposição aos valores indetectáveis encontrados nas linfadenopatias inflamatórias ou de origem não tireoidiana (26). Esta observação tem sido confirmada em vários estudos (8,23-27,31). Em 1999, Frasodalti e cols. mostraram que a combinação da citologia com a Tg-PAAF permitiu a detecção de linfonodos metastáticos com sensibilidade maior que a de cada técnica isoladamente (25). O mesmo grupo demonstrou, recentemente, que a associação da dosagem de Tg-PAAF ao exame citológico aumentou a sensibilidade deste último de $84,8 \%$ para $95,6 \%(8)$.

Na casuística de 44 linfonodos visualizados à US cervical de pacientes com carcinoma papilífero, acompanhados no ambulatório de tireóide da EPM/UNIFESP (27), 29/44 apresentaram dosagem de Tg-PAAF indetectável $(<1,0 \mathrm{ng} / \mathrm{mL})$, em concordância com a citolo- gia "reacional", e 15/44 apresentaram dosagem de TgPAAF elevada (tabela 5). Destes pacientes, 1.315 apresentavam citologia compatível com metástases de carcinoma papilífero, porém em 2/15, apesar das dosagens de Tg-PAAF elevadas (50 e $1.500 \mathrm{ng} / \mathrm{mL}$, respectivamente), a citologia foi não-diagnóstica (material insuficiente para análise) (tabela 5 , pacientes 1 e 8 ). Nestes 2 casos, a Tg-PAAF foi fundamental para o diagnóstico de metástase de carcinoma de tireóide. Em todos os casos com TgPAAF elevada, o diagnóstico de metástases foi confirmado pelo exame histopatológico. Neste estudo, a realização da citologia permitiu o diagnóstico de linfonodo reacional ou metastático em 95\% dos pacientes, com sensibilidade de $86,5 \%$ e especificidade de $100 \%$. Porém, se ao estudo citológico acrescentarmos a dosagem de TgPAAF, a sensibilidade passa a ser de $100 \%$.

Em pacientes tireoidectomizados, os dados da literatura são concordantes que, na ausência de tecido tireoidiano, os linfonodos devem apresentar níveis indetectáveis de Tg-PAAF (8,23-27). Recentemente, Boi e cols. alertaram para a dosagem de Tg-PAAF em linfonodos de pacientes não tireoidectomizados. Nestes, os autores estabeleceram um cut-off para que a dosagem de Tg-PAAF fosse discriminatória entre linfonodos reacionais e metastáticos (32). Porém, novos estudos são necessários para melhor avaliação do comportamento da Tg-PAAF em linfonodos destes pacientes.

Portanto, a dosagem da Tg-PAAF deve ser realizada em conjunto com o estudo citológico obtido da

Tabela 5. Descrição dos 11 pacientes (15 linfonodos) com metástases cervicais (27).

\begin{tabular}{|c|c|c|c|c|c|c|c|}
\hline Paciente & $\begin{array}{l}\text { sTg-LT } \\
\text { (ng/mL) }\end{array}$ & $\begin{array}{l}\text { sTg- } \\
\text { hipo }\end{array}$ & AcATg & Citologia & $\begin{array}{l}\text { Tg-PAAF } \\
\text { (ng/mL) }\end{array}$ & Dose / PCI & $\begin{array}{c}\text { Diam mm / } \\
\text { Nível }\end{array}$ \\
\hline 1 & $<1,0$ & 2,1 & Pos & ND & 50 & $100 \mathrm{mCi} / \mathrm{Neg}$ & $8 /$ III \\
\hline 2 & $<1,0$ & 21,5 & $\mathrm{Neg}$ & Meta & 15.600 & $30 \mathrm{mCi} / \mathrm{NR}$ & 9 / III \\
\hline 3 & $<1,0$ & 9,8 & Neg & Meta & 258 & $100 \mathrm{mCi} / \mathrm{LT}$ & 12 / III \\
\hline 4 & $<1,0$ & 2,5 & Neg & Meta & 4.000 & $100 \mathrm{mCi} / \mathrm{Neg}$ & $20 /$ IV \\
\hline 5 & $<1,0$ & 38 & Neg & Meta & $>1.000$ & $250 \mathrm{mCi} / \mathrm{LT}+$ pulmão & $9 / \mathrm{VI}$ \\
\hline 6 & $<1,0$ & 1,2 & $\mathrm{Neg}$ & Meta & $>1.000$ & $250 \mathrm{mCi} / \mathrm{LT}$ & $6 / \mathrm{VI}$ \\
\hline $7 \mathrm{~A}$ & 1,8 & NR & Neg & Meta & 2.949 & $100 \mathrm{mCi} /$ Linf & 13 / IVE \\
\hline 7B & 1,8 & NR & Neg & Meta & 3.400 & $100 \mathrm{mCi} /$ Linf & 30 / IVD \\
\hline 7C & 1,8 & NR & $\mathrm{Neg}$ & Meta & 14.257 & $100 \mathrm{mCi} / \operatorname{Linf}$ & $15 /$ IVE \\
\hline 8 & 3,2 & NR & Neg & ND & 1.500 & $200 \mathrm{mCi} /$ Linf & 12 / IV \\
\hline 9 & 3,9 & NR & $\mathrm{Neg}$ & Meta & 17.700 & $5 \mathrm{mCi} / \mathrm{Neg}$ & 14 / VI \\
\hline 10 & 5,9 & NR & Neg & Meta & 1.500 & $100 \mathrm{mCi} / \mathrm{Neg}$ & 17 / IV \\
\hline $11 \mathrm{~A}$ & 3,8 & NR & $\mathrm{Neg}$ & Meta & $>1.000$ & $250 \mathrm{mCi} / \mathrm{LT}$ & $32 / 11$ \\
\hline $11 \mathrm{~B}$ & 3,8 & NR & Neg & Meta & $>1.000$ & $250 \mathrm{mCi} / \mathrm{LT}$ & 20 / III \\
\hline $11 \mathrm{C}$ & 3,8 & NR & Neg & Meta & $>1.000$ & $250 \mathrm{mCi} / \mathrm{LT}$ & 12 / IV \\
\hline
\end{tabular}

sTg-LT: dosagem de sTg em vigência de Levo-tiroxina, sTg hipo: dosagem de sTg em vigência de TSH > $30 \mathrm{mUl} / \mathrm{ml}$, AcATg: anticorpos anti-tiroglobulina, Tg-PAAF: dosagem de Tg no lavado da agulha da PAAF, PCl: pesquisa de corpo inteiro, ND: não diagnóstica, Meta: metástase de carcinoma de tiróide, Neg: Negativo, NR: não realizado, LT: leito tiroidiano, Linf: linfonodo, D: direita, E: esquerda. 
PAAF e está indicada no seguimento de pacientes com carcinoma de tireóide (principalmente no carcinoma papilífero) tratados com tireoidectomia total, cuja US evidencia linfonodo "suspeito". Outros estudos são necessários em pacientes não tireoidectomizados.

\section{Proposta de abordagem de pacientes com lin- fonodos aparentes na US}

Uma proposta de abordagem de pacientes com carcinoma diferenciado de tireóide e linfadenopatia cervical é apresentada na figura 5.

Metástases de carcinoma papilífero podem apresentar crescimento lento. Assim, a permanência das características da linfadenopatia na US não exclui malignidade, sendo a regressão indicador de benignidade. O período de seis meses para uma nova avaliação nos parece bastante seguro, pois o possível impacto do atraso no diagnóstico de metástases sobre a perspectiva de cura parece ocorrer apenas com intervalo maior que um ano (33).

\section{ULTRA-SONOGRAFIA AUXILIANDO O TRATAMENTO DE METÁSTASES CERVICAIS}

Em casos selecionados, metástases cervicais isoladas podem ser tratadas com escleroterapia com etanol ou ablação com radiofreqüência. Nestas situações, a US é fundamental tanto na orientação da injeção intranodal do etanol $(34,35)$ quanto no posicionamento adequado dos eletrodos para ablação por radiofreqüência (36). Com a detecção precoce de metástases cervicais pela US e pelo prognóstico favorável, é provável que terapias alternativas ganhem mais espaço, especialmente a alcoolização $(34,35)$, poupando os pacientes dos riscos da cirurgia e dos efeitos adversos do radioiodo.

\section{LIMITAÇÕES DA US EM PACIENTES COM CÂNCER DE TIREÓIDE}

O compartimento cervical central (nível VI) constitui um sítio comum de metástases em pacientes com carcinoma papilífero, e sua abordagem inicial de rotina ainda é motivo de controvérsias. Uma das limitações da US é sua baixa sensibilidade na avaliação pré-operatória deste compartimento $(37,38)$.

US tem sido recomendada após a tireoidectomia e antes da ablação com ${ }^{131}$ I com a finalidade de mensurar os remanescentes tireoidianos e avaliar a persistência de metástases linfonodais $(4,15)$. No entanto, alterações pós-cirúrgicas persistem por até 3 meses (15) e impedem uma avaliação ultra-sonográfica adequada, não sendo recomendada a US precocemente após a tireoidectomia.

Alguns pacientes apresentam micrometástases cervicais detectadas apenas na US (exame clínico normal, PCI negativa e Tg indetectável). Vale ressaltar que a história natural dessas metástases ainda é desconhecida, sendo possível que muitas destas não apresentem nenhuma progressão em pacientes de baixo risco mantidos sob adequada supressão do TSH (39).

Finalmente, a US é um método conhecidamente examinador-dependente, sendo possível que a

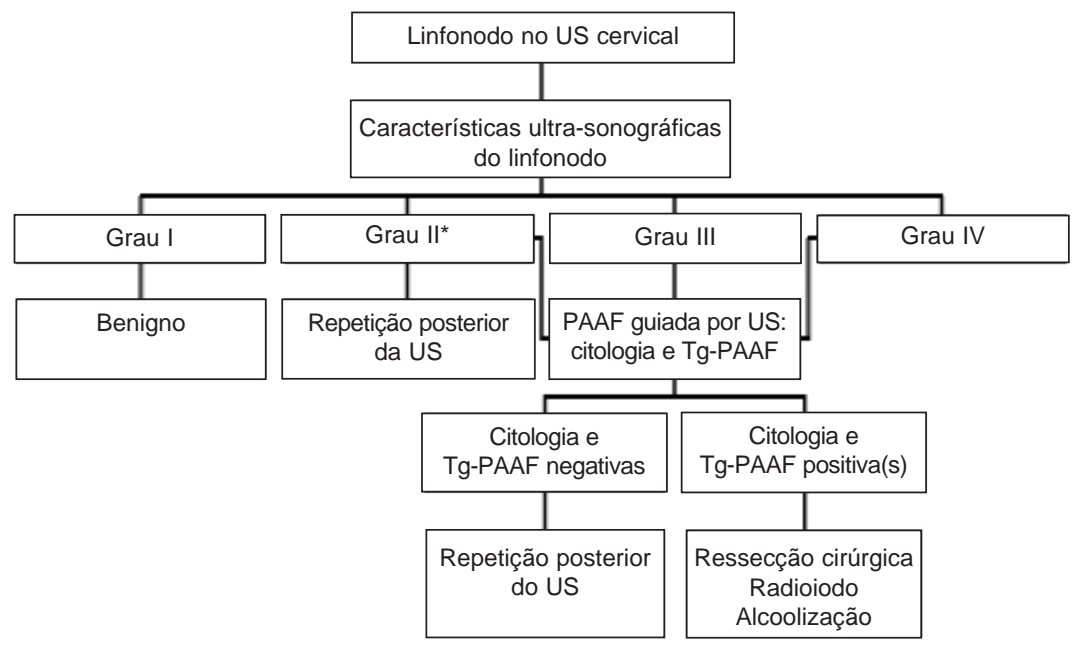

Figura 5. Abordagem dos pacientes com carcinoma de tireóide e linfonodos aparentes na US cervical. 
sensibilidade encontrada em alguns estudos não seja facilmente reproduzida, pela dependência do examinador. Além disso, observações e/ou descrições inadequadas das características ultra-sonográficas, base para conduta clínica em pacientes com linfonodomegalia cervical, podem causar iatrogenias, como PAAF e repetições de exames de imagem desnecessariamente. Desta forma, o ideal para o seguimento dos pacientes com câncer diferenciado da tiróide seria o estabelecimento de uma prática onde os endocrinologistas referissem os pacientes apenas para radiologistas e citologistas experientes nas especificidades e novos recursos destas técnicas, apesar de o nosso sistema de saúde muitas vezes não favorecer esta prática.

\section{REFERÊNCIAS}

1. Cooper DS, Doherty GM, Haugen BR, Kloos RT, Lee SL, Mandel SJ, et al. Management guidelines for patients with thyroid nodules and differentiated thyroid cancer. Thyroid 2006; 16:109-42.

2. Pacini $F$, Schlumberger $M$, Dralle $H$, Elisei $R$, Smit JWA, Wiersinga W, et al. European consensus for the management of patients with thyroid carcinoma of the follicular epithelium. Eur J Endocrinol 2006;154:787-803.

3. Schlumberger MJ. Diagnostic follow-up of well-differentiated thyroid carcinoma: historical perspective and current status. J Endocrinol Invest 1999;22:S3-7.

4. Pacini F. Follow-up of differentiated thyroid cancer. Eur $\mathbf{J}$ Nucl Med Mol Imaging 2002;29:S492-6.

5. Torlontano M, Attard M, Crocetti U, Tumino S, Bruno R, Costante G, et al. Follow-up of low-risk patients with papillary thyroid cancer: role of neck ultrasonography in detecting lymph node metastases. J Clin Endocrinol Metab 2004;89:3402-7.

6. Pacini F, Molinaro E, Castagna MG, Agate L, Elisei R, Ceccarelli $C$, et al. Recombinant human thyrotropin-stimulated serum thyroglobulin combined with neck ultrasonography has the highest sensitivity in monitoring differentiated thyroid carcinoma. J Clin Endocrinol Metab 2003;88:3668-73.

7. Rosário PW, Fagundes TA, Maia FF, Franco A, Figueiredo MB, Purisch S. Ultrasonography in the diagnosis of cervical recurrence in patients with differentiated thyroid carcinoma. J Ultrasound Med 2004;23:915-20.

8. Frasoldati A, Pesenti M, Gallo M, Caroggio A, Salvo D, Valcavi R. Diagnosis of neck recurrences in patients with differentiated thyroid carcinoma. Cancer 2003;97:90-6.

9. Schlumberger M, Berg G, Cohen O, Duntas D, Jamar F, Jarzab B, et al. Follow-up of low-risk patients with differentiated thyroid carcinoma: an European perspective. Eur J Endocrinol 2004;150:105-12.

10. Rosário PW, Borges MA, Fagundes TA, Franco AC, Purisch S. Is stimulation of thyroglobulin $(\mathrm{Tg})$ useful in low-risk patients with thyroid carcinoma and undetectable $\mathrm{Tg}$ on thyroxin and negative neck ultrasound? Clin Endocrinol (Oxf) 2005;62:121-5

11. David A, Blotta A, Rossi R, Zatelli MC, Bondanelli M, Roti E, et al. Clinical value of different responses of serum thyroglobulin to recombinant human thyrotropin in the follow-up of patients with differentiated thyroid carcinoma. Thyroid 2005;15:158-64.

12. Torlontano M, Crocetti U, D'Aloiso L, Bonfitto N, Di Giorgio A, Modoni S, et al. Serum thyroglobulin and 131 | whole body scan after recombinant human TSH stimulation in the followup of low-risk patients with differentiated thyroid cancer. Eur J Endocrinol 2003;148:19-24.
13. Rosário PW, Cardoso LD, Fagundes TA, Barroso AL, Padrão $\mathrm{EL}$, Rezende LL, et al. Revisiting serum thyroglobulin in the follow-up of patients with differentiated thyroid carcinoma. Arq Bras Endocrinol Metab 2004;48:480-6.

14. Kouvaraki MA, Shapiro SE, Fornage BD, Edeiken-Monro BS, Sherman SI, Vassilopoulou-Sellin R, et al. Role of preoperative ultrasonography in the surgical management of patients with thyroid cancer. Surgery 2003;134:946-54; discussion 954-5.

15. Rosário PW, Barroso AL, Padrão EL, Maia FF, Purisch S, Fagundes TA, et al. Thyroglobulin before ablation and correlation with posttreatment scanning. Laryngoscope 2005:114:264-7.

16. Mazzaferri EL. Empirically treating high serum thyroglobulin levels. J Nucl Med 2005;46:1079-88.

17. Rosário PW, Faria S, Bicalho L, Gatti Alves MF, Borges MA, Purisch $\mathrm{S}$, et al. Ultrasonographic differentiation between metastatic and benign lymph nodes in patients with papillary thyroid carcinoma. J Ultrasound Med 2005;24:1385-9.

18. Ahuja A, Ying M, King W, Metreweli C. A practical approach to ultrasound of cervical lymph nodes. J Laryngol Otol 1997; 111:245-56

19. Ahuja AT, Chow L, Chick W, King W, Metreweli C. Metastatic cervical nodes in papillary carcinoma of the thyroid: ultrasound and histological correlation. Clin Radiol 1995;50:229-31.

20. Kessler A, Rappaport Y, Blank A, Marmor S, Weiss J, Graif M. Cystic appearance of cervical lymph nodes is characteristic of metastatic papillary thyroid carcinoma. J Clin Ultrasound 2003;31:21-5.

21. Giuffrida D, Santonocito MG, lurato MP, Freni V, Ippolito A, Squatrito S. Echography at "high resolution" in the diagnosis of cervical lymphadenopathies in follow-up of thyroid carcinoma. Minerva Endocrinol 1997;22:61-6.

22. Ahuja A, Ying M. An overview of neck node sonography. Invest Radiol 2002;37:333-42.

23. Cignarelli M, Ambrosi A, Marino A, Lamacchia O, Campo M, Picca G, et al. Diagnostic utility of thyroglobulin detection in fine-needle aspiration of cervical cystic metastatic lymph nodes from papillary thyroid cancer with negative cytology. Thyroid 2003;12:1163-7.

24. Baskin HJ. Detection of recurrent papillary thyroid carcinoma by thyroglobulin assessment in the needle washout after fine-needle aspiration of suspicious lymph nodes. Thyroid 2004:14:959-63.

25. Frasoldati A, Toschi E, Zini M, Flora M, Caroggio A, Dotti C, et al. Role of thyroglobulin measurement in fine-needle aspiration biopsies of cervical lymph nodes in patients with differentiated thyroid cancer. Thyroid 1999:9:105-11.

26. Pacini F, Fugazzola L, Lippi F, Ceccarelli C, Centoni R, Miccoli $P$, et al. Detection of thyroglobulin in fine needle aspirates of nonthyroidal neck masses: a clue to the diagnosis of metastatic differentiated thyroid cancer. J Clin Endocrinol Metab 1992; 74:1401-4.

27. Biscolla RP, Ikejiri ES, Mamone MC, Nakabashi CCD, Andrade VP, Kasamatsu TS, et al. Diagnóstico de metástases de carcinoma diferenciado de tiróide através da dosagem de tiroglobulina no líquido obtido da lavagem da agulha utilizada na punção aspirativa. Arq Bras Endocrinol Metab 2007:51/3: in press.

28. Boland GW, Lee MJ, Mueller PR, Mayo-Smith W, Dawson SL, Simeone JF. Efficacy of sonographically guided biopsy of thyroid masses and cervical lymph nodes. AJR Am J Roentgenol 1993;161:1053-6.

29. Rodriguez JM, Reus M, Moreno A, Martinez M, Soria T, Carrasco L, et al. High-resolution ultrasound associated with aspiration biopsy in the follow-up of patients with differentiated thyroid cancer. Otolaryngol Head Neck Surg 1997;117:694-7.

30. Krishnamurthy S, Bedi DG, Caraway NP. Ultrasound-guided fine-needle aspiration biopsy of the thyroid bed. Cancer 2001;93:199-204.

31. Uruno T, Miyauchi A, Shimizu K, Tomoda C, Takamura Y, Ito $Y$, et al. Usefulness of thyroglobulin measurement in fineneedle aspiration biopsy specimens for diagnosing cervical lymph node metastasis in patients with papillary thyroid cancer. World J Surg 2005;29:483-5. 
32. Boi F, Baghino G, Atzeni F, Lai ML, Faa G, Mariotti S. The diagnostic value for differentiated thyroid carcinoma metastases of thyroglobulin ( $\mathrm{Tg}$ ) measurement in washout fluid from fine-needle aspiration biopsy of neck lymph nodes is maintained in the presence of circulating anti-Tg antibodies. J Clin Endocrinol Metab 2006;91:1364-9.

33. Mazzaferri EL, Kloos R. Current approaches to primary therapy for papillary and follicular thyroid cancer. J Clin Endocrinol Metab 2001;86:1447-63.

34. Lewis BD, Hay ID, Charboneau JW, Mclver B, Reading CC, Goellner JR. Percutaneous ethanol injection for treatment of cervical lymph node metastases in patients with papillary thyroid carcinoma. AJR Am J Roentgenol 2002;178:699704.

35. Bianchini EX, Ikejiri ES, Mamone MC, Paiva ER, Maciel RMB, Furlanetto RP. Injeção percutânea de etanol no tratamento de nódulos tiroidianos sólidos, císticos e autônomos. Arq Brasil Endocrinol Metab 2003;47:543-51.

36. Dupuy DE, Monchik JM, Decrea C, Pisharodi L. Radiofrequency ablation of regional recurrence from well-differentiated thyroid malignancy. Surgery 2001;130:971-7.

37. King AD, Ahuja AT, To EW, Tse GM, Metreweli C. Staging papillary carcinoma of the thyroid: magnetic resonance imaging vs. ultrasound of the neck. Clin Radiol 2000;55:222-6.
38. Ito $Y$, Tomoda C, Uruno T, Takamura Y, Miya A, Kobayashi K, et al. Clinical significance of metastasis to the central compartment from papillary microcarcinoma of the thyroid. World J Surg 2006;30:91-9.

39. Borges MA, Rosário PW, Valadão MM, Costa GC, Purisch S. Metástases linfonodais detectadas apenas ultra-som. Qual o significado clínico? Arq Bras Endocrinol Metab 2006;50:S655.

\section{Endereço para correspondência:}

Pedro Weslley Souza Rosário

Centro de Estudos e Pesquisa da Clínica de

Endocrinologia e Metabologia (CEPCEM)

Av. Francisco Sales 1111, 5० andar, Ala D

30150-221 Belo Horizonte, MG

Fax: (31) 3213-0836

E-mail: pedrorosario@globo.com 